\title{
Permeability of the small intestine to substances of different molecular weight
}

C. A. LOEHRY, A. T. R. AXON ${ }^{1}$, P. J. HILTON, R. C. HIDER, AND B. CREAMER

From St Thomas' Hospital, London

SUMMARY The permeability of the rabbit small intestine has been studied by measuring the plasma clearances of water-soluble molecules over the molecular weight range $60-33,000$. An inverse relationship has been demonstrated between permeability and molecular weight.

The significance of these findings in relation to current concepts of the 'pore hypotheses' is discussed, and the possible physiological and pathological implications are considered.

\section{Part 1}

Substances are continually passing from the body through the small bowel wall and into the lumen. Most of them come directly or indirectly from plasma either passing in some way through the epithelium or by incorporation into cells and later lost with them into the lumen. Some substances will be secreted by goblet cells and Paneth cells.

In previous experiments (Loehry, Croft, Singh, and Creamer, 1969), which were done to study the mechanism of intestinal excretion of iron and vitamin $B_{12}$, it was found that two peaks of intestinal excretion occurred after the parenteral administration of a radioactive tracer. The first peak, which occurred shortly after the injection of the tracer, corresponded to the period of time when the concentration of the tracer in the plasma was high. The second peak, which occurred at 36-42 hours, corresponded with the period of time during which cells containing the tracer exfoliated from the tips of the villi. The first type of excretion appears to be related to plasma concentration and is unlikely to be due to a glandular mechanism; it is probably the result of the tracer exuding across the intestinal wall.

In the following experiments an attempt was made to quantitate this exudative loss, relating ${ }^{1}$ Correspondence: Dr A. T. R. Axon, Gastrointestinal Laboratory St Thomas' Hospital, London, SE1.

Received for publication 27 November 1969. the amount of substance excreted to its concentration in the plasma and deriving from this relationship a plasma clearance value. Plasma clearance values have been calculated for a variety of substances, and a relationship has been shown to exist between the clearance values and the molecular weights. The substances tested were chosen primarily to give a range of molecular weights and most of them are not actively handled by the small intestine.

\section{METHODS}

Albino rabbits weighing $2 \cdot 2-2 \cdot 6 \mathrm{~kg}$ were used, the experiments being performed under intravenous nembutal anaesthesia.

The external jugular vein on one side was cannulated for intravenous infusions and the carotid artery on the other side was similarly cannulated for the collection of blood samples. The abdomen was opened and both kidney pedicles were tied off in order to prevent the loss of injected substances in the urine. The jejunum was cannulated just distal to the ligament of Treitz and just proximal to the ileocaecal valve. After preliminary perfusions to clear the gut of faecal residues, the small intestine was perfused with physiological normal saline at $40^{\circ} \mathrm{C}$ at a rate of $600 \mathrm{ml} /$ hour.

Substances to be tested were injected into the external jugular vein and after a 10-minute equilibration period a blood sample was with- 


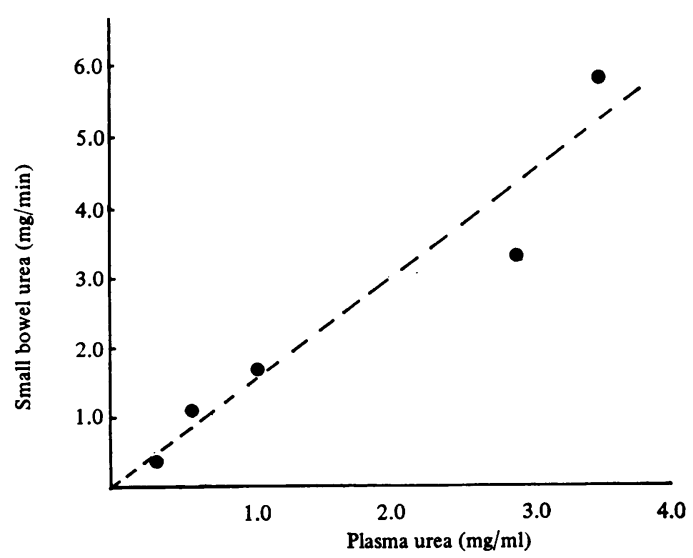

Fig. 1 The relationship between the amount of urea excreted by the small bowel and the plasma concentration of urea.

\begin{tabular}{llc}
\hline Substance & Clearance $($ ml/min $)$ & Molecular Weight \\
\hline Urea & $1 \cdot 8$ & 60 \\
Creatinine & $0 \cdot 51$ & 113 \\
Fructose & $0 \cdot 31$ & 180 \\
Vitamin $B_{12}$ & 0.053 & 1,000 \\
Inulin & 0.028 & 5,500 \\
PVP & 0.007 & 33,000 \\
\hline
\end{tabular}

Table Intestinal clearance values

drawn from the opposite carotid artery and intestinal perfusate was collected for a timed 20-minute period. At the end of this time a second blood sample was taken. The quantity of substance in the 20-minute perfusate was estimated, and this value gave the amount excreted by the intestine in that time. The two blood levels were averaged to estimate the plasma level of the substance during the 20-minute period. Two blood samples were not taken in every experiment as it was found that in some cases the plasma levels varied to a negligible extent; in these cases one sample at 10 minutes was taken. In the case of fructose the plasma levels fell so rapidly that it was necessary continuously to infuse the jugular vein with fructose solution. Several 20-minute tests were run in succession on each animal, increasing the plasma concentration of the substances each time.

The following materials and methods in the experiments were used: urea, estimated on the AutoAnalyzer using the Berthelot method; creatinine, estimated on the AutoAnalyzer by the alkaline picrate method. Inulin was estimated on the AutoAnalyzer using a modification of the Dawborn method; the blank subtraction was made by measuring in the hydrolysed and unhydrolysed states, and it was found necessary to concentrate the perfusate in some of the experi-

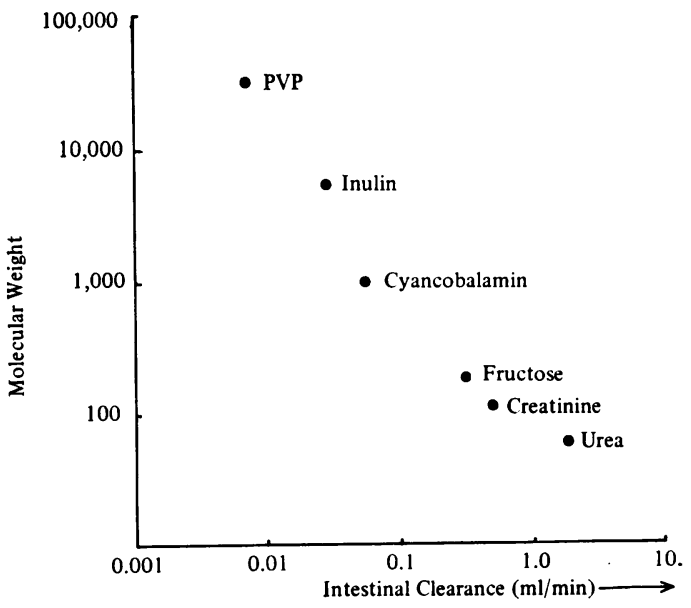

Fig. 2 The relationship between the logarithm of the molecular weight and the logarithm of the intestinal excretion.

ments in order to gain an accurate estimation. Fructose estimation was performed by a modification of the above technique. ${ }^{125}$ I-labelled polyvinylpyrrolidone (PVP) and ${ }^{68} \mathrm{Co}-$ labelled cyanocobalamin were measured by scintillation counter and scaler. The doses of vitamin $B_{12}$ used were of such a magnitude as to saturate the protein-binding capacity of the plasma and the results in this paper are thought to relate to unbound molecules of $B_{12}$.

\section{RESULTS}

Figure 1 shows a graph derived from a typical experiment, in this case, urea. As the plasma concentration of urea rises, so its intestinal excretion increases in direct proportion.

A convenient way of expressing the ability of a system to excrete a substance is to use the concept of 'plasma clearance'. The plasma clearance of a substance is defined as 'that volume of plasma from which all the test substance has been removed in one minute' and can be calculated as follows:

$$
\text { Clearance }=\frac{\text { amount of substance excretion } / \mathrm{min}}{\text { concentration of that substance in }}
$$

It can be seen that this formula is represented in Fig. 1 where the amount of urea excreted/min is plotted against its plasma concentration. The plasma clearance remained constant over the range of plasma concentrations studied and corresponds to the slope of the line.

A variety of substances was chosen for study and their molecular weights varied widely. Intestinal clearance values were ascertained in each case and the results are shown in the Table where it can be seen that smaller molecules are excreted at a much higher rate than large 


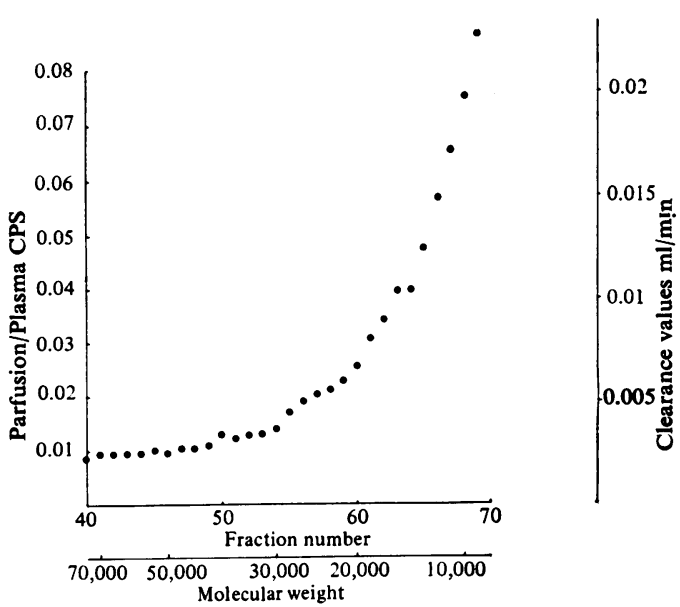

Fig. 3 Blood-to-bowel lumen PVP experiment: the relationship between the molecular weights of $P V P$ fractions and the perfusate/plasma concentration ratios. Molecular weights approximating to the fraction numbers are on the lower scale, and calculated clearance values are shown on the right.

ones. Figure 2 is a $\log$ molecular weight/log intestinal clearance graph and shows a linear relationship. Most substances behaved like urea, the clearance remaining constant over a wide range of plasma concentrations; glucose was an exception, however, for, as its concentration increased, its excretion failed to rise proportionately, ie, its plasma clearance fell. It was thought that this might be the result of glucose being actively reabsorbed, and so the experiment was repeated, this time including phlorizin in the perfusate to inhibit the transport mechanism. This modification did not alter the excretion pattern.

\section{Part II}

In the first part of this paper, it has been shown that under experimental conditions substances may pass from the plasma into the small intestinal lumen at a rate inversely proportional to their molecular weights. During the course of these studies, PVP labelled with ${ }^{125} \mathrm{I}$ was shown to cross the small intestinal wall from plasma to lumen in small but measurable quantities. Polyvinylpyrrolidone is a synthetic polymer with a nominal molecular weight range from 8,000 to 80,000 which is available commercially from the Radiochemical Centre, Amersham, Bucks, labelled with ${ }^{125} \mathrm{I}$. The mixture of polymer molecules can be fractionated according to their molecular size by filtration on a column of Sephadex G200. By analysing the PVP which has passed across the intestinal wall and comparing individual fractions with corresponding fractions of the original solution, the relative permeability of the small intestinal wall to different sized macromolecules can be determined. In the present series of experiments permeability in relation to molecular size was studied from the plasma to the gut lumen and also from the lumen to the plasma.

\section{MATERIALS AND METHODS}

Studies were performed on five adult rabbits weighing $2 \cdot 0-2 \cdot 6 \mathrm{~kg}$. The animals were prepared for the experiments as described in the first part of this paper. In the first group of experiments (two rabbits) the small intestine was perfused with normal saline at body temperature, $100 \mu \mathrm{C}$ of ${ }^{125} \mathrm{I}$-labelled PVP was injected intravenously and after a 15-minute equilibration period plasma and perfusate samples were collected. In the second group of experiments (three rabbits) the small intestine was perfused with normal saline to which had been added $100 \mu \mathrm{C}$ of ${ }^{125}$ I-labelled polyvinylpyrrolidone. The perfusate was continuously recycled for 90 minutes after which the plasma sample was taken.

In both groups the plasma and perfusate samples were subjected to gel filtration analysis using the method described by Hardwicke, Hulme, Jones, and Ricketts (1968). Five $\mathrm{ml}$ of the sample to be analysed was placed on a $76 \times 2 \mathrm{~cm}$ column of Sephadex G200 and eluted with 1\% saline to which had been added $10 \mathrm{mg} \%$ sodium azide. Four ml fractions were collected automatically and the radioactivity of each fraction was measured in a well type scintillation counter. To ensure correct alignment of corresponding plasma and perfusate samples, bovine serum albumin was added to the perfusate before analysis and the peak albumin concentration in the eluate was determined by continuous monitoring of the absorption of ultraviolet light at wavelength $280 \mathrm{~m} \mu$. In order to obtain adequate radioactivity in the perfusate samples in group I, preliminary concentration was effected by freeze drying.

The Sephadex column used in these experiments was calibrated by measuring the elution volumes of various proteins of known molecular weight: IGG $(150,000)$, serum albumin $(68,000)$, ovalbumin $(44,000)$, myoglobin $(18,000)$, and insulin $(6,000)$. The elution volume was proportional to the logarithm of the molecular weight over the range studied.

\section{RESULTS}

The results obtained in the two experiments in group I were virtually identical. Figure 3 shows the relationship in one experiment between the 


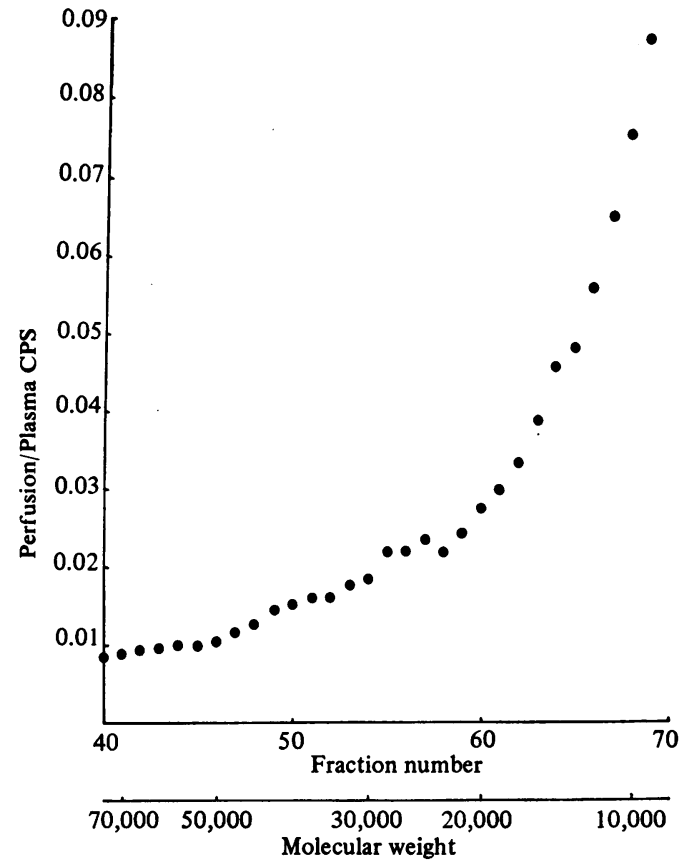

Fig. 4 Bowel-lumen-to-blood PVP experiment: the relationship between the molecular weights of $P V P$ fractions and plasma/perfusate concentration ratios (cf Fig. 3).

perfusate/plasma activity ratio of PVP and the fraction number. The ratio is lowest in the early fractions representing the high molecular weight polymers and increases progressively with successive fractions, ie, the smaller molecules of PVP were cleared more rapidly than the large ones. Molecular weights appropriate to various fractions derived from the calibration of the column have been included in the figure.

In the second group of experiments, an attempt was made to compare the permeability of the small intestinal wall to PVP from the lumen to the plasma with the results obtained in the reverse direction. The results in three separate studies bore a close similarity to each other, a typical example of the relationship between the plasma/perfusate activity ratio and the fraction number being shown in Figure 4. Since the plasma/perfusate ratio rises continuously throughout these experiments it is inappropriate to express the results in terms of a clearance. However, there is a close similarity between the results obtained in these experiments and those in group I.

These studies indicate that water-soluble substances in the molecular weight range 8,00080,000 may pass through the small intestinal wall at a rate dependent on their molecular size. The permeability pattern is virtually identical whether diffusion is taking place from plasma to lumen or from lumen to plasma.

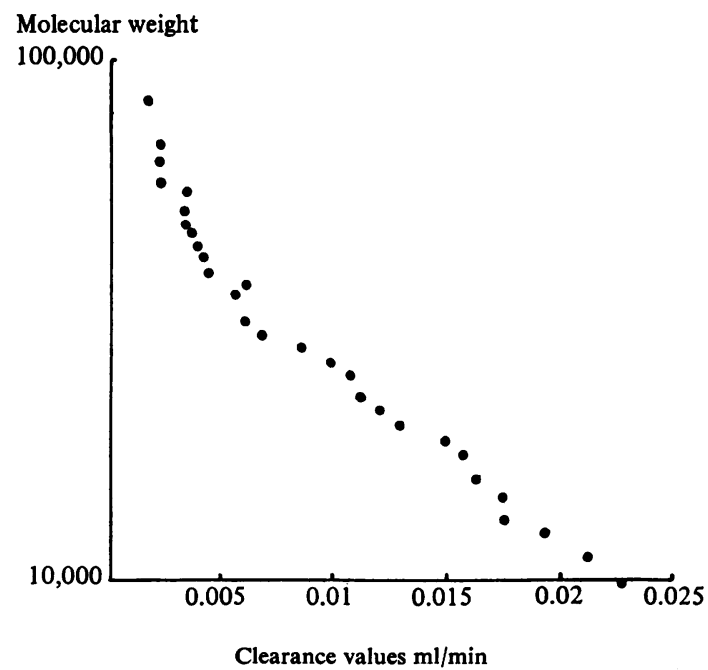

Fig. 5 Log/log graph showing the relationship between the intestinal clearance of PVP fractions and molecular weight.

\section{Discussion}

For many years it has been known that the permeability of the small intestine to different substances varies enormously, depending upon the nature of the substance tested. Fat-soluble substances can cross the small intestinal wall with facility even when the molecular weight is comparatively large. On the other hand, the permeability of the small intestine to watersoluble substances is largely dependent upon molecular size; substances with a molecular weight of over 180 are said hardly to penetrate at all. These findings have given rise to the 'pore hypothesis' which postulates that the mucosal wall is limited by a fat-soluble membrane perforated at intervals by water-filled pores having a diameter estimated as lying between 3 and 8.8 $\AA$ (Fordtran, Rector, Maynard, Soter, and Kinney, 1965).

Nevertheless, it has been known for some time that water-soluble substances with a molecular weight much in excess of 180 can penetrate the intestinal wall. Albumin and PVP are known to pass from the body into the small intestinal lumen, and the above experiments confirm that PVP with an effective molecular weight of 33,000 can pass both from the intestinal lumen into the plasma and in the opposite direction. To explain this phenomenon, one must postulate that pores with a much greater 
diameter than $9 \AA$ exist or alternatively that a different mechanism of permeation has been studied.

This paper has set out to examine the rate of permeation of substances across the bowel wall, and Fig. 2 shows that there is a linear relationship between log molecular weight and $\log$ clearance. This relationship holds for molecular weights between 60 and 33,000 and experiments designed to test this relationship for very high molecular weights $(8,000$ to 80,000$)$ show that clearance values for these behave in a similar way (Fig. 5). These findings suggest that the permeating mechanism is the same for large molecules as for small ones, otherwise one might expect to see a cut-off phenomenon above a certain molecular size. If the pore hypothesis is correct, the data could be accounted for by postulating a normal distribution of pore size, ie, a very few large pores, a moderate number of medium-sized pores, and very many small pores.

The site of the porous barrier is not known; it may be at any point between the capillary wall and the intestinal lumen or it may be a combination of several overlapping barriers. Permeating molecules have first to transude across the capillary wall, which, in the small intestine, comprises a continuous epithelium perforated by intracellular fenestra which are closed by a diaphragm of cell membrane and surrounded by a continuous basement membrane (Bennet, Luft, and Hampton, 1959). Substances are believed to cross the capillary wall by passing through pores in the fenestra, the diameters of which are thought to vary from 70 to $90 \AA$ for small pores to $500 \AA$ for large ones. Ferritin has been shown to cross the capillary wall and the basement membrane of the capillary does not appear to present a barrier (Clementi and Palade, 1969). It is unlikely, therefore, that the capillary wall represents an important impediment to the permeation of the substances studied above.

Having passed the basement membrane of the small intestine, three possible routes are open to the permeating molecule: they may pass out either between the mucosal cells, or at the tips of the villi through the gap left by exfoliating cells, or they may pass through the mucosal cells themselves, leaking from the epithelial border into the bowel lumen. If exuding molecules made their exit between cells or from gaps in the mucosa one would not expect there to be any difference in excretion pattern between metabolically active and inactive molecules, but it has been shown above, and by other workers (Cocco and Hendrix, 1965), that glucose behaves differently, and this evidence supports the view that the route followed is through the mucosal cell.

It may be helpful to think in human terms when trying to assess the importance of this exudative loss. If the rabbit figures are converted to human terms, the urea clearance by the small intestine would be $50 \mathrm{ml} / \mathrm{min}$, which represents a daily excretion of $20 \mathrm{~g}$ of urea. Most of the exuding substances are reabsorbed under normal conditions but in patients who have diarrhoea or malabsorption this exudation may make an appreciable contribution to faecal losses. In some conditions where mucosal anatomy and physiology are distorted exudation may be increased. It is interesting to note that biologically important molecules tend to be protein bound in the plasma, thereby increasing their effective molecular weight and reducing the rate at which they can pass out of the body.

We wish to thank the Clinical Chemistry Laboratory for performing various chemical estimations and the Isotope Laboratory for the use of their equipment. We also thank Miss J. Cole for her technical assistance for which we were very grateful.

\section{References}

Bennet, H. S., Luft, J. H., and Hampton, J. C. (1959). Morphological classifications of vertebrate capillaries. Amer. $J$. Physiol., 196, 381-390.

Clementi, F., and Palade G. E. (1969). Intestinal capillaries I. J. Cell Biol., 41, 33-58.

Cocco, A. E., and Hendrix, T. R. (1965). The effect produced by Phlorizin and hypertonic saline on sugar movement from blood to intestinal lumen. Bull. Johns Hopk. Hosp., 117, 296-305.

Fordtran, J. S., Rector, F. C., Maynard, F. E., Soter, N., and Kinney,J.(1965). Permeability characteristics of the human small intestine. J. clin. Invest., 44, 1935-1944.

Hardwicke, J., Hulme, B., Jones, J. H., and Ricketts, C. R. (1968). Measurement of glomerular permeability to polydispersed radioactivity labelled macromolecules in normal rabbits. Clin. Sci., 34, 505-514.

Loehry, C. A., Croft, D. N., Singh, A. K., and Creamer, B. (1969) Cell turnover in the rat small intestinal mucosa: An appraisal of cell loss. Gut, 10, 13-18. 